\title{
Nicotine Modulates Reorienting of Visuospatial Attention and Neural Activity in Human Parietal Cortex
}

\author{
Christiane M Thiel*, 1,2, Karl Zilles ${ }^{1,2,3}$ and Gereon R Fink ${ }^{1,2,4}$ \\ IInstitute of Medicine, Research Centre Jülich, Jülich, Germany; ${ }^{2}$ Brain Imaging Centre West, Research Centre Jülich, Jülich, Germany; ${ }^{3} \mathrm{C}$ and $\mathrm{O}$ \\ Vogt Institute for Brain Research, Heinrich-Heine-University, Düsseldorf, Germany; ${ }^{4}$ Department of Neurology, University Hospital RWTH \\ Aachen, Aachen, Germany
}

\begin{abstract}
Prior studies in animals and humans indicate that reorienting of visuospatial attention is modulated by the cholinergic agonist nicotine. We have previously identified neural correlates of alerting and reorienting attention in humans and found that the parietal cortex is specifically involved in reorienting. This study investigates whether the alerting and reorienting systems, especially in the parietal cortex, are modulated by nicotine. We used event-related functional magnetic resonance imaging ( $\mathrm{MMRI}$ ) and studied I 5 nonsmoking volunteers under placebo and nicotine (NICORETTE ${ }^{\circledR}$ polacrilex gum I and $2 \mathrm{mg}$ ). Subjects performed a cued target detection task with four different types of randomly intermixed trials (no, neutral, valid, and invalid cue trials). Alerting was captured by comparing BOLD activity and reaction times (RTs) in neutrally cued trials with no cue trials. Reorienting was isolated by comparing invalidly with validly cued trials On the behavioral level, nicotine affected reorienting of attention by speeding RTs in invalidly cued trials; alerting was not affected by nicotine. Neurally, however, nicotine modulated both attentional systems. Pharmacologic effects on alerting-related brain activity were mainly evident as modulation of BOLD responses in the right angular gyrus and right middle frontal gyrus due to a reduction of neural activity in no cue trials. In the reorienting system, effects of nicotine were mainly evident in the left intraparietal sulcus and precuneus and due to a reduction of neural activity in invalidly cued trials. We conclude that nicotine enhances reorienting of attention in visuospatial tasks and that one behavioral correlate of speeded RTs is reduced parietal activity.
\end{abstract}

Neuropsychopharmacology (2005) 30, 810-820, advance online publication, 19 January 2005; doi: I 0. I 038/s..npp. 1300633

Keywords: magnetic resonance imaging; functional; acetylcholine; attention; nicotine; parietal lobe

\section{INTRODUCTION}

Cued target detection tasks provide a simple means to study the subcomponents of selective attention and their pharmacological modulation. One of these functional components is called 'alerting' and defines a phasic state of general readiness and increased responsiveness to targets that follow the presentation of a warning cue. Alerting is captured by comparing neutrally cued trials with no cue trials (eg Coull et al, 2001; Fan et al, 2002; Witte and Marrocco, 1997). Another component which can be investigated with this task is attentional 'reorienting'. Reorienting is necessary when misleading advance information is provided in the form of a spatially invalid cue and can be captured by comparing invalidly with validly cued

\footnotetext{
*Correspondence: Dr CM Thiel, Institute of Medicine, Research Centre Jülich, 52425 Jülich, Germany, Tel: + 492461612483 , Fax: + 49 246| 6I 2820, E-mail: c.thiel@fz-juelich.de

Received 3 August 2004; revised 22 October 2004; accepted 26 October 2004

Online publication: 2 November 2004 at http://www.acnp.org/citations/ Npp I I 0204040353/default.pdf
}

trials (eg Fan et al, 2002; Witte et al, 1997). Alerting and reorienting correlate with neural activity in different brain regions (Thiel et $a l, 2004$ ) and can be dissociated pharmacologically by showing that some drugs affect alerting and leave reorienting intact while others act in the opposite way.

Evidence in rats, monkeys, and humans suggests that the neurotransmitter acetylcholine (ACh) is involved specifically in reorienting of visuospatial attention, while the noradrenergic system is involved in alerting (Posner and Fan, 2004). Cholinergic basal forebrain nuclei provide the major source of cholinergic input to the cerebral cortex. If these nuclei are lesioned, monkeys are impaired in detecting stimuli following invalid cues (Voytko, 1996). Conversely, increased cholinergic activity, achieved, for example, by systemic administration of the cholinergic agonist nicotine, was shown to speed reaction times (RTs) to invalidly cued stimuli (Witte et al, 1997; Stewart et al, 2001; Phillips et al, 2000). This indicates impaired reorienting of visuospatial attention with cholinergic depletion and improved reorienting with cholinergic stimulation, and contrasts with the role of noradrenergic neurotransmission, which is hypothesized to contribute to alerting but not to reorienting 
(Witte and Marrocco, 1997; Clark et al, 1989; see, however, Coull et al, 2001).

Behavioral data suggest that human smokers benefit from cholinergic stimulation in situations which require reorienting of attention. For example, Witte et al (1997) compared RTs in smokers who smoked a cigarette and nonsmokers and found faster responses to invalidly cued trials in smokers as compared to nonsmokers. Further evidence for improved reorienting with nicotine comes from a study which showed that smokers improved their efficiency to respond to invalid trials immediately after smoking a cigarette as compared to 1 or $24 \mathrm{~h}$ later (Murphy and Klein, 1998). Whether similar benefits are also observed in nonsmoking subjects, and can thus be ascribed to the primary effect of nicotine rather than a reversal of withdrawal-induced deficits, is controversial. Griesar et al (2002) found no evidence for improved attentional reorienting after nicotine in nonsmoking subjects who received a nicotine patch.

Given the evidence for a specific role of nicotinic cholinergic neurotransmission in reorienting of attention, it is of further interest to know which brain areas mediate such effects. There is preliminary evidence obtained from rats, where nicotine was infused locally into the posterior parietal cortex, which indicates that the effect of nicotine in visually cued target detection tasks is mediated by the drugs' action on cholinergic receptors in the parietal cortex (Beane et al, 2002), a region that plays a crucial role in attentional reorienting in humans (Thiel et al, 2004; Corbetta et al, 2000; Petersen et al, 1989).

Here, we investigate in nonsmoking subjects whether a nicotine-induced improvement of attentional reorienting is associated with modulation of parietal activity. We combined event-related fMRI with the administration of nicotine or placebo, and used a cued target detection task with valid, invalid, neutral, and no cue trials to study reorienting of visuospatial attention as well as alerting. Our hypothesis was that nicotine would specifically affect behavioral measures, that is, RTs to invalid trials, and neural measures of reorienting, that is, parietal activity.

\section{METHODS}

\section{Subjects}

A total of 19 right-handed nonsmokers (8 males, 11 females; age range: $20-31$ years, mean: 23.8 years) with no history of acute or chronic medical disease gave informed consent to participate in the study. No subject was on medication (except for contraceptives). All subjects had normal, or corrected to normal, vision. A clinical evaluation was first carried out to ensure that subjects had no conditions contraindicative for nicotine administration. Ethics approval was obtained from the ethics committee of the University of Düsseldorf. Nonsmokers were used to avoid confounding effects of nicotine abstinence on cognitive effects, that is, the possibility of reversing a deprivationinduced attentional deficit, rather than increasing attentional processes per se. No subject had used nicotine during the last 2 years and most subjects $(n=17)$ had never smoked regularly at all. Subjects were asked to abstain from alcohol $12 \mathrm{~h}$ before each session and from caffeine $3 \mathrm{~h}$ prior to testing. Four volunteers were excluded from further analysis due to excessive head movement $(n=3)$ or technical failure $(n=1)$, leaving 15 subjects whose data were analyzed.

\section{Drug Administration}

We used a within-subjects design. Each subject was tested on three experimental sessions, separated by at least 1 week. The order of drug administration was counterbalanced over subjects. Nicotine was delivered in the form of a polacrilex gum (NICORETTE ${ }^{\circledR}$ mint taste, Pharmacia) in 1 and $2 \mathrm{mg}$ doses and a chewing gum with mint taste as placebo. Subjects were asked to chew the gum for $30 \mathrm{~min}$ at a rate of one chew per $3 \mathrm{~s}$. Scanning started immediately after chewing had finished. In nonsmokers, nicotine plasma levels are on average $1.3 \mathrm{ng} / \mathrm{ml}$ at this time point (Heishman and Henningfield, 2000). The half-life of nicotine is about $2 \mathrm{~h}$ (Benowitz et al, 1988).

\section{Stimuli and Experimental Paradigm}

We used a cued target detection task with central predictive cuing (Posner, 1980; Thiel et al, 2004). Stimuli were projected onto a screen in front of the participant in the MRI scanner. Viewing distance was approx. $29 \mathrm{~cm}$. The baseline display was composed of a central diamond $\left(1.3^{\circ}\right.$ eccentric in each visual field) and two peripheral boxes $\left(3^{\circ}\right.$ wide and $9.6^{\circ}$ eccentric in each visual field). The cue stimulus consisted of the central diamond brightening for $100 \mathrm{~ms}$. The target stimulus was either a filled diamond or a filled circle $\left(1.3^{\circ}\right.$ wide) and appeared for $100 \mathrm{~ms}$ in one of the peripheral boxes. The cue-target interval was 400 or $700 \mathrm{~ms}$ to reduce temporal orienting. We used four trial types with different cueing conditions (see Figure 1). In

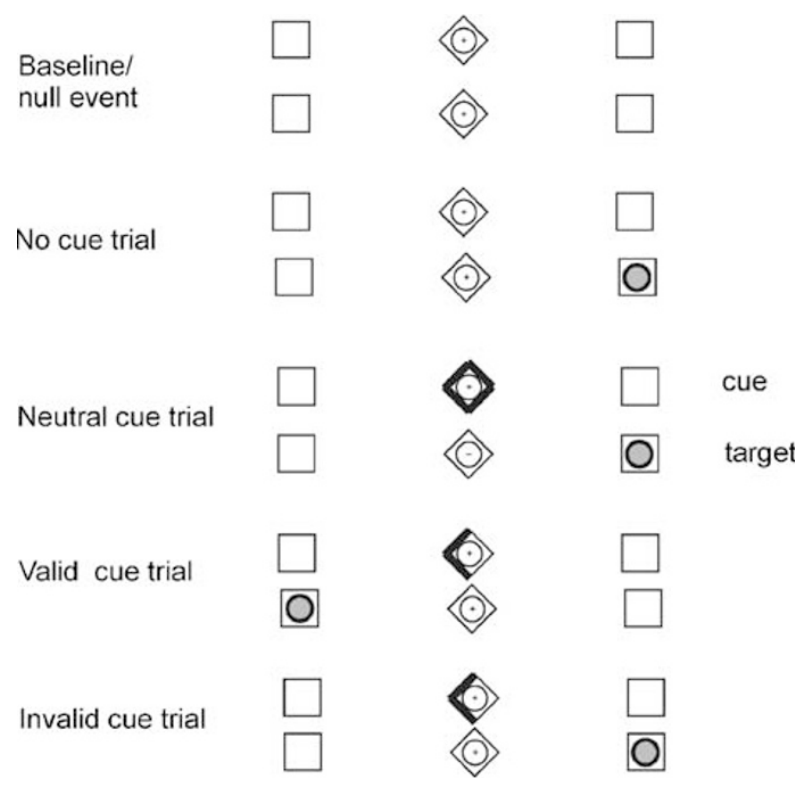

Figure I Experimental paradigm. Illustration of different trial types. Alerting was captured by comparing cerebral activity in neutral cue trials with no cue trials. Reorienting was isolated by subtracting cerebral activity in invalid cue trials from valid cue trials. 
trials with a spatial cue, the left or right side of the central diamond brightened for $100 \mathrm{~ms}$, indicating the side of subsequent target appearance. In $80 \%$ of these spatial trials the target appeared on the side indicated by the cue (validly cued trials), in $20 \%$ of the cases the target appeared on the opposite side (invalidly cued trials). In neutral cue trials, the diamond brightened as a whole, giving no spatial information about where the target would appear. In no cue trials the central diamond remained unchanged, giving no indication that a target would appear subsequently. Additionally, $6 \%$ of all trials were catch trials in which a spatial cue but no target appeared. Trials were presented every $2 \mathrm{~s}$. The order of trial types was randomized, as was the occurrence of left and right targets and cue-target intervals. The total number of events was 504, with 192 validly cued trials, 48 invalidly cued trials, 48 neutral trials, 48 no cue trials, 24 catch trials, and 144 'null events' (Josephs and Henson, 1999), where a baseline stimulus was displayed. The use and random inclusion of null events lead to variable trial-onset asynchronies. Subjects were instructed to maintain fixation throughout the experiment and to covertly detect any peripheral target as fast as possible. Volunteers made responses with the right index finger on a button of a keypad placed on the right side of their body. Prior to scanning, subjects were informed about the different trial types. They were told that spatial cues were highly informative and encouraged to use these cues to improve task performance. A 2 min training was performed before each scanning session.

\section{Data Acquisition}

A SONATA MRI system (Siemens, Erlangen, Germany) operating at $1.5 \mathrm{~T}$ was used to obtain $\mathrm{T} 2{ }^{*}$-weighted echoplanar images (EPI) with blood oxygenation leveldependent $(B O L D)$ contrast (matrix size: $64 \times 64$, pixel size: $3.12 \times 3.12 \mathrm{~mm}^{2}$ ). In all, 411 volumes of 244 -mm-thick axial slices were acquired sequentially with a $0.8 \mathrm{~mm}$ gap (repetition time $=2.5 \mathrm{~s}$, echo time $=66 \mathrm{~ms}$ ). The first 5 volumes were discarded to allow for $\mathrm{T} 1$ equilibration effects. Images were spatially realigned to the first volume to correct for interscan movement. The time series of each voxel was realigned temporally to the middle slice to correct for differences in slice acquisition time. Images were normalized to a standard EPI template (resampled to $3 \times 3 \times 3 \mathrm{~mm}^{3}$ voxel). The data were then smoothed with a Gaussian kernel of $8 \mathrm{~mm}$ full-width-half-maximum to accommodate intersubject anatomical variability.

\section{Statistical Analyses of Imaging Data}

Data were analyzed with Statistical Parametric Mapping software SPM2, Wellcome Department of Imaging Neuroscience, London (http://www.fil.ion.ucl.ac.uk/spm2.html; Friston et al, 1995), employing a random effects model. At the first level, all the three sessions (ie placebo, nicotine $1 \mathrm{mg}$, and nicotine $2 \mathrm{mg}$ ) were incorporated into one design matrix. For each session, 10 event types were defined. These consisted of nine effects of interest (no cue right target, no cue left target, neutral cue right target, neutral cue left target, valid cue right target, valid cue left target, invalid cue right target, invalid cue left target, and catch trial) and one effect of no interest (missed responses). The event types were time-locked to the onset of cue by a canonical synthetic hemodynamic response function (HRF) and its first-order temporal derivative. With a cue-target interval of 400 or $700 \mathrm{~ms}$, both cue- and target-related activity will contribute to the signal. The six head movement parameters (three rigid body translations and rotations) were included as confounds. Data were globally scaled to 100 across scans and highpass-filtered at $1 / 128 \mathrm{~Hz}$. The parameter estimates for the canonical HRF and linear contrasts of these estimates comprised the data for the second stage of analyses.

We focused on contrasts isolating alerting (neutral-no cue trials) and reorienting of attention (invalid-valid cue trials) within each group and between groups. Alerting and reorienting were first estimated within each group (ie placebo, nicotine $1 \mathrm{mg}$, and nicotine $2 \mathrm{mg}$ ). Significant differences between placebo and drug were then estimated using the following four planned contrasts testing for increases and decreases of neural activity with nicotine: (i) a contrast which isolates brain regions showing greater alerting effects under placebo as compared to nicotine (placebo(neutral-no cue)-0.5(nicotine $1 \mathrm{mg}$ (neutral-no cue) + nicotine $2 \mathrm{mg}($ neutral-no cue))); (ii) a contrast which captures the reverse, that is, brain areas with greater alerting effects under nicotine as compared to placebo (0.5(nicotine $1 \mathrm{mg}$ (neutral-no cue) + nicotine $2 \mathrm{mg}$ (neutral-no cue))-placebo(neutral-no cue)); (iii) a contrast showing greater reorienting effects under placebo as compared to nicotine (placebo(invalid-valid cue)-0.5(nicotine $1 \mathrm{mg}$ (invalid-valid cue) + (nicotine $2 \mathrm{mg}$ (invalid-valid cue)) ), and (iv) the reverse contrast which isolates brain areas with higher reorienting activations under nicotine as compared to placebo ( 0.5 (nicotine $1 \mathrm{mg}$ (invalid-valid cue) + nicotine 2 mg(invalid-valid cue))-placebo(invalid-valid cue)). We restricted our analysis to brain regions showing a main effect of task (ie all trial types $v s$ baseline display). For this purpose, the relevant contrasts of parameter estimates from the 15 subjects were entered into an ANOVA (without constant term) with nonsphericity correction and were estimated in a conjunction with the main effect. All activations and figures reported are at a level of significance of $p<0.0001$ and a cluster threshold of more than five contiguous voxels. The level of significance of $p<0.0001$ was chosen to correct for multiple contrasts (ie 10). The reported coordinates correspond to the standard Montreal Neurological Institute (MNI) brain. Activations are displayed on a coregistered and normalized structural T1 image of a single volunteer for illustrative purposes using the software mricro (Rorden and Brett, 2000). The effect maxima of drug by condition interactions were plotted as a function of trial type and analysed with post hoc Tukey tests.

\section{Statistical Analyses of Behavioral Data}

Median RTs were calculated for each trial type and drug condition in each subject. The means of median RTs were analyzed with two repeated-measures ANOVAs, with factors alerting and drug (collapsed over 1 and $2 \mathrm{mg}$ ) and reorienting and drug, respectively (in analogy to the imaging contrasts). Since prior behavioral studies all 
indicate increased reorienting with nicotine, $p$-values are reported on a one-sided significance level.

\section{Subjective and Physiological Measures}

In every session, subjective drug effects were repeatedly assessed with visual analogue scales (Bond and Lader, 1974). Rating scores were grouped into the three factors 'alertness', 'contentedness', and 'calmness', according to Bond and Lader (1974). Mean scores were analyzed for drug effects with an ANOVA for repeated measures. Other bodily symptoms were assessed with a symptoms checklist. The pulse was checked before the start of the scanning session and analyzed for drug effects with an ANOVA for repeated measures and a contrast testing for a linear trend. Throughout the experiment, pulseoximetry was performed. After the experiment, volunteers were asked to fill in a questionnaire assessing, among others, general task difficulty and difficulty of different trial types (on a scale from 1 to 7 ).

\section{RESULTS}

\section{Behavior: RT Data}

Figure 2a illustrates the mean RTs and standard errors of mean (SEM) for all trials and drug conditions. Descriptively, both doses of nicotine speeded RTs in all trials; this effect was, however, the biggest for invalid trials. To investigate nicotine's effects on alerting and reorienting statistically, two within-subject ANOVAs testing for differences in alerting and reorienting were performed on mean RTs. These tested for differences in alerting (ie neutral as compared to no cue trials) and reorienting (ie invalid as compared to valid trials) under nicotine as compared to placebo. There was a trend for nicotine affecting reorienting $(T(14)=1.68, p=0.057)$, while alerting was not influenced by nicotine $(T(14)=1.24, p=0.116)$. The number of responses to catch trials (false alarms) did not differ between drug groups, and were 1.0, 0.87, and 1.07 for placebo, and both nicotine doses, respectively.

Since there is evidence from other neurotransmitter systems that effects of pharmacological stimulation depend on initial performance (Mattay et al, 2000; Mehta et al, 2000) and since we only had a tendency for behavioral effects of nicotine on reorienting, we performed a further ANOVA including the size of the validity effect (ie invalid-valid RT) under placebo as covariate. This yielded a significant reorienting by drug interaction $(\mathrm{F}(1,13)=8.00, p=0.014)$ and a reorienting by drug by validity effect interaction $(\mathrm{F}(1,13)=15.05, p=0.002)$. In order to illustrate this finding, Figure $2 \mathrm{~b}$ shows the effects of nicotine on those volunteers who show a big validity effect under placebo and those who show a small validity effect under placebo $(n=7$ each; groups obtained by a median split). The results demonstrate that only those subjects who have a big validity effect (ie are slow in reorienting their attention) benefit from nicotine and that these benefits are stronger with the higher dose.

\section{Behavior: Subjective and Physiological Measures}

Nicotine increased the pulse rate dose dependently (placebo: $69.7 \pm 2.7$ mean \pm SEM, nicotine $1 \mathrm{mg}$ : $71.6 \pm 1.8$, nico-
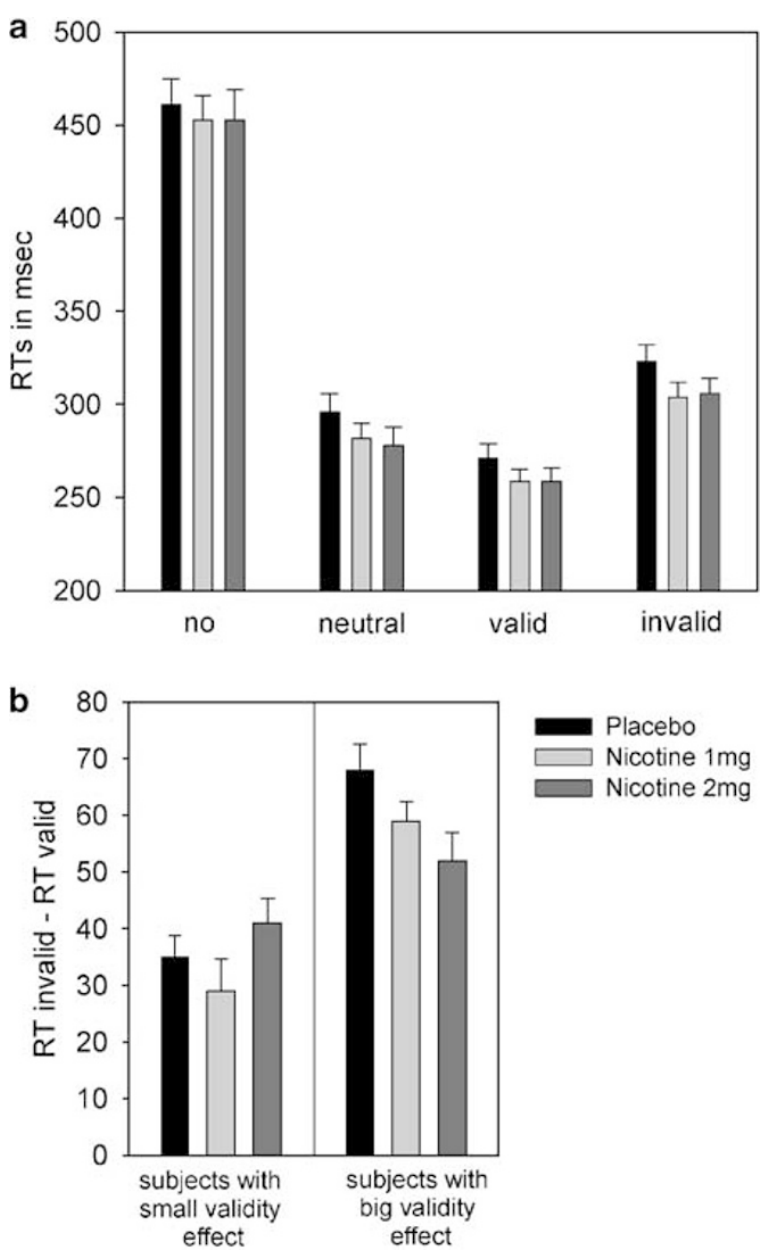

Figure 2 Behavioral data. (a) Mean reaction times (RT in ms) and standard errors (SEM) as a function of trial type and drug condition. (b) Nicotinic modulation of validity effects (RT invalid-RT valid) depending on performance under placebo.

tine $2 \mathrm{mg}: 74.9 \pm 2.7$; specific contrast testing a linear trend: $\mathrm{F}(1,13)=9.06, p=0.01)$. There were no effects of nicotine on subjective ratings of alertness, contentedness or calmness before or after scanning (all $p>0.2$ ). There were also no drug effects on task difficulty; under all conditions, subjects perceived the task as easy. At the end of the three sessions, subjects were asked to indicate on which session they got nicotine or placebo. All subjects correctly indicated the nicotine sessions.

\section{fMRI: Global Signal}

A potential confound in drug fMRI studies is the effect that a drug might have on global blood flow. To investigate whether nicotine might have interfered with global cerebral blood flow ( $\mathrm{gCBF}$ ), we compared the estimated global BOLD signal across the brain between the placebo and drug sessions. No differences in this global estimate were evident (ANOVA $\mathrm{F}(2,16)=0.043 ; p=0.95)$.

\section{fMRI Data Alerting}

The alerting effect was captured by comparing BOLD activity in neutral cue trials with no cue trials (see 
Figure 3). Using this contrast, neural activity was mainly evident in bilateral extrastriate regions under placebo with peaks in right fusiform gyrus, left middle occipital, and left middle temporal gyrus $(x=42, y=-69, z=-24 ; Z=7.0$; 393 voxels; $x=-42, y=-63, z=-15 ; Z=5.11 ; 94$ voxels, $x=-54, y=-69, z=-3 ; Z=4.21 ; 18$ voxels). These brain regions were also significantly activated under 1 and $2 \mathrm{mg}$ of nicotine ( $1 \mathrm{mg}: x=42, y=-69, z=-24 ; Z=7.34 ; 231$ voxels; $x=-42, y=-78, z=-18 ; Z=4.94 ; 73$ voxels; $2 \mathrm{mg}$ : $x=42, y=-69, z=-24 ; Z=5.91 ; 283$ voxels; $x=-42$, $y=-66, z=-12 ; Z=5.19 ; 50$ voxels, $x=-39, y=-81$, $z=-30 ; Z=4.85 ; 20$ voxels). Additionally, there was a significant right posterior parietal activation encompassing superior and inferior aspects of the angular gyrus $(x=30$, $y=-72, z=-54 ; Z=4.52 ; 8$ voxels; $x=54, y=-54, z=24$; $Z=4.51 ; 13$ voxels) under $2 \mathrm{mg}$ of nicotine. Similar activations were present under $1 \mathrm{mg}$ of nicotine $(x=57$, $y=-54, z=27 ; Z=4.16 ; 3$ voxels; $x=30, y=-75, z=48$ $Z=3.78 ; 2$ voxels), but did not survive the voxel threshold.

A direct comparison between nicotine and placebo testing for increased alerting activity with nicotine revealed an alerting by drug interaction in the right angular gyrus/ intraparietal sulcus, right middle frontal gyrus, bilateral right superior frontal gyrus, and left cerebellum (see Table 1 and Figure 4a). These regions showed higher activity during alerting with nicotine. The right parts of Figure $4 \mathrm{~b}$ and $c$ illustrate that the drug by condition interaction in the
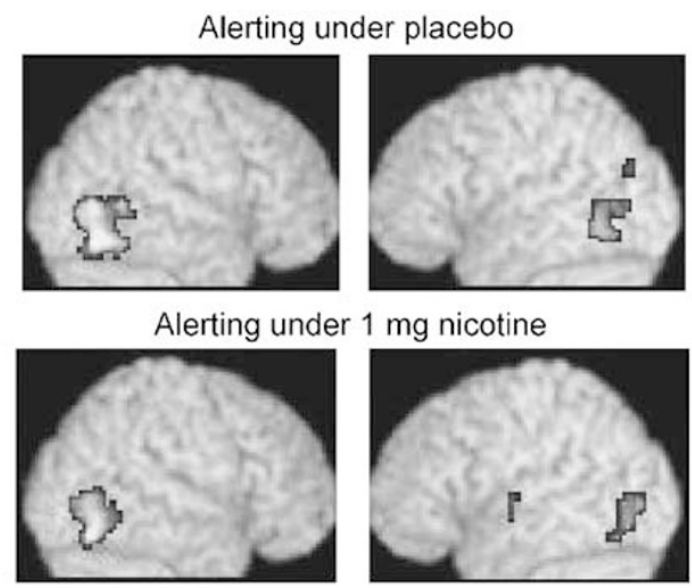

Alerting under $2 \mathrm{mg}$ nicotine
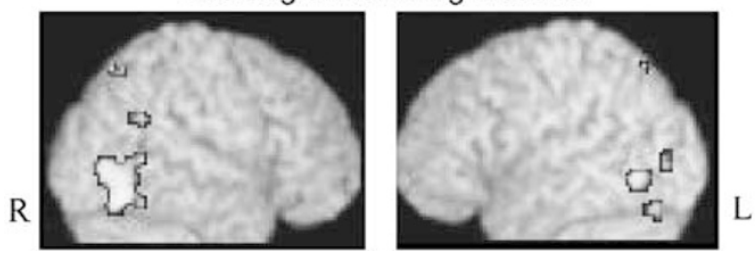

Figure 3 Alerting under placebo and nicotine. Brain regions showing higher responses to neutral as compared to no cue trials. All activations $(p<0.000 \mathrm{I}$, uncorrected) are shown on a normalized structural image of a single volunteer. Note that activations close to the midline are not displayed. Extensive activations with peaks in the right fusiform gyrus and left middle occipital gyrus were seen under placebo. The I mg nicotine condition shows activations in the right fusiform gyrus and left middle occipital gyrus, and subcortically in the left globus pallidus. Under $2 \mathrm{mg}$ nicotine, activation peaks were found in the right fusiform gyrus, left middle occipital gyrus, and right angular gyrus. angular gyrus and middle frontal gyrus is mainly driven by a strong reduction in neural activity after nicotine in no cue trials. The opposite contrast, which isolates higher neural

Table I Higher Activations under Nicotine as Compared to Placebo for Alerting

\begin{tabular}{|c|c|c|c|c|c|c|}
\hline Region & Side & $x$ & $y$ & $\mathbf{z}$ & Voxels & Z-score \\
\hline \multicolumn{7}{|l|}{ Parietal } \\
\hline Angular gyrus/intraparietal sulcus & $\mathrm{R}$ & 39 & -48 & 45 & 90 & 5.23 \\
\hline \multicolumn{7}{|l|}{ Frontal } \\
\hline Middle frontal gyrus & $\mathrm{R}$ & 51 & 12 & 42 & 31 & 4.71 \\
\hline Superior frontal gyrus & & 0 & 12 & 66 & | | & 4.31 \\
\hline Cerebellum & $L$ & -36 & -63 & -42 & 12 & 4.42 \\
\hline
\end{tabular}
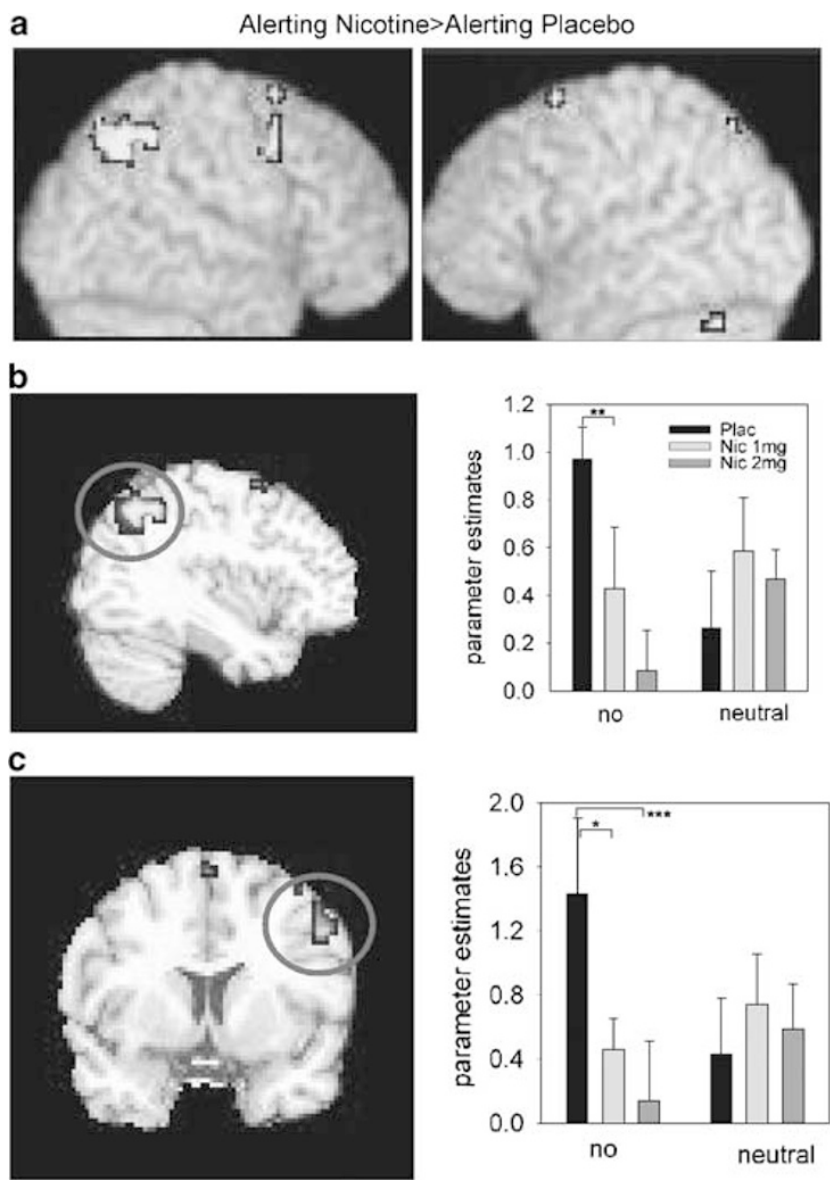

Figure 4 Alerting by drug interaction. (a) Brain regions showing higher responses to neutral as compared to no cue trials under nicotine as compared to placebo (see also Table I). Note the significantly increased activity in right angular gyrus/intraparietal sulcus and middle and superior frontal gyrus. (b) Right angular gyrus activation displayed on a sagittal section with a plot of effect size (mean and SEM) as a function of trial type and drug. Data are taken from the maximally activated voxel of the circled region. (c) Coronal view of frontal activations. The plot illustrates the middle frontal activity as a function of trial type and drug. ${ }^{*} p<0.05$; *** $p<0.01$; **** $p>0.001$ (post hoc Tukey's tests). 
Table 2 Higher Activations under Placebo as Compared to Nicotine for Alerting

\begin{tabular}{|c|c|c|c|c|c|c|}
\hline Region & Side & $x$ & $y$ & $\mathbf{z}$ & Voxels & Z-score \\
\hline \multicolumn{7}{|l|}{ Occipital } \\
\hline Lateral occ.-temp. sulcus & $\mathrm{L}$ & -45 & -60 & -12 & 10 & 4.34 \\
\hline \multirow[t]{2}{*}{ Lingual gyrus } & $\mathrm{R}$ & 18 & -60 & -18 & 16 & 4.54 \\
\hline & $\mathrm{R}$ & 3 & -72 & -15 & 7 & 4.34 \\
\hline \multirow[t]{2}{*}{ Cerebellum } & $\mathrm{R}$ & 3 & -60 & -27 & 21 & 4.95 \\
\hline & L & -21 & -66 & -33 & 11 & 4.30 \\
\hline
\end{tabular}

activity with alerting under placebo as compared to nicotine, yielded increased activations in the left lateral occipito-temporal sulcus and right lingual gyrus and bilateral cerebellum (see Table 2). The left lateral occipitotemporal sulcus is the anterior part of the left extrastriate region showing significant alerting effects in the placebo condition.

A final comparison tested the dose-dependent effects of nicotine by directly comparing alerting-related activity under both doses of nicotine. The $2 \mathrm{mg}$ dose significantly increased alerting-related activity in the left thalamus $(x=-18, y=-21, z=12 ; Z=5.23 ; 11$ voxels), left cingulate sulcus $(x-9=, y=6, z=39 ; Z=5.15 ; 11$ voxels), right cerebellum $(x=9, y=-57, z=-12 ; Z=4.56 ; 17$ voxels; $x=33, y=-63, z=-36 ; Z=4.52 ; 13$ voxels), and left superior colliculus $(x=-6, y=-30, z=-3 ; Z=4.39 ; 8$ voxels) when compared to the $1 \mathrm{mg}$ dose. There were no regions significantly more active under $1 \mathrm{mg}$ of nicotine as compared to $2 \mathrm{mg}$ of nicotine. Note that parietal and frontal activations were not dependent on the dose of nicotine used (even when less conservative statistical testing, such as that on the parameter estimates plotted in Figure $4 \mathrm{~b}$ and $c$, was performed).

\section{fMRI Data: Reorienting}

Neural correlates of reorienting were identified by contrasting invalid cue trials with valid cue trials (see Figure 5). Under placebo, this contrast yielded activations in the left intraparietal sulcus/angular gyrus $(x=-45, y=-48, z=51$; $Z=4.77 ; 29$ voxels), left supramarginal gyrus $(x=-33$, $y=-69, z=51 ; Z=4.27 ; 29$ voxels), and precuneus $(x=0$, $y=-63, z=45 ; Z=4.47 ; 19$ voxels). There was also neural activity in the right intraparietal sulcus; the extent of this activity did, however, not exceed five contiguous voxels. Higher frontal activity to invalid as compared to valid trials was evident in the left and right middle frontal gyrus $(x=-48, y=6, z=42 ; Z=4.76 ; 19$ voxels; $x=30, y=-3$, $z=54 ; Z=4.76 ; 19$ voxels). Under $1 \mathrm{mg}$ of nicotine, reorienting-related activity was only evident in the right middle frontal gyrus $(x=33, y=-3, z=54 ; Z=4.72 ; 35$ voxels). Under $2 \mathrm{mg}$ of nicotine, increased neural activations to invalid as compared to valid trials were found in several frontal and temporal brain regions and in the cerebellum and thalamus $(x=0, y=-54, z=-12 ; Z=4.94$;

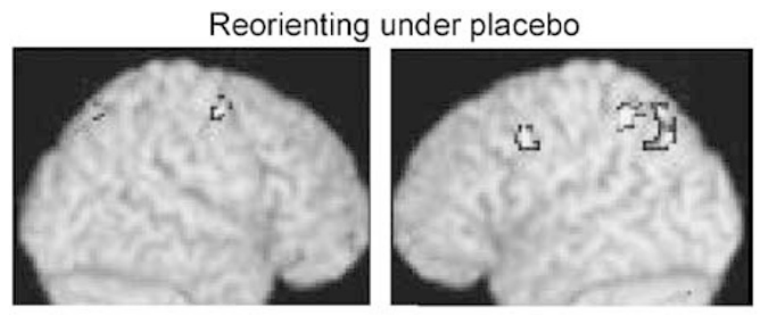

\section{Reorienting under $1 \mathrm{mg}$ nicotine}

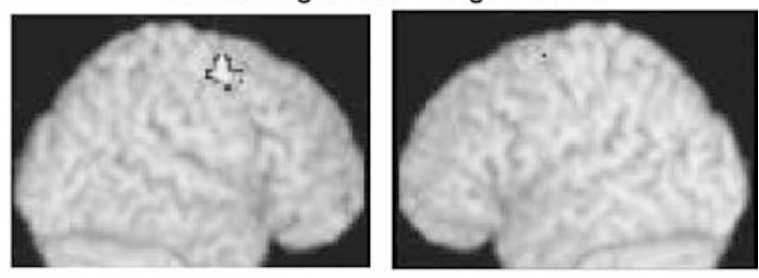

Reorienting under $2 \mathrm{mg}$ nicotine

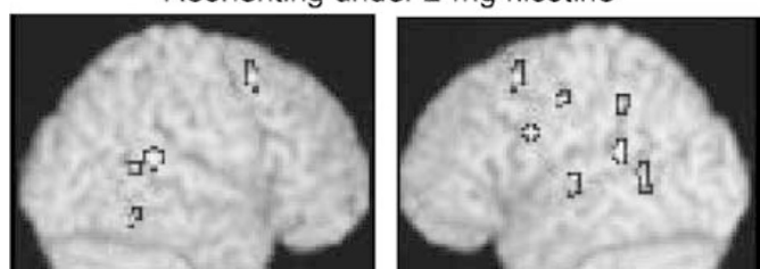

Figure 5 Reorienting under placebo and nicotine. Brain regions showing higher responses to invalid as compared to valid trials. Under placebo, reorienting attention significantly increased activity in the left parietal and bilateral frontal regions (intraparietal sulcus/angular gyrus, precuneus, middle frontal gyrus). With I mg of nicotine the right middle frontal gyrus was significantly activated. With $2 \mathrm{mg}$ of nicotine, increased neural activity was evident in several frontal (right superior frontal gyrus, left inferior precentral sulcus) and temporal brain areas (left and right superior temporal gyrus).

17 voxels; $x=-18, y=-15, z=-3 ; Z=4.92 ; 8$ voxels). The frontal activity was found medial and inferior to the activity reported under placebo and was located in right superior frontal gyrus ( $x=9, y=15, z=54 ; Z=4.85 ; 11$ voxels $)$ and left inferior precentral sulcus $(x=-54, y=6, z=24$; $Z=4.17 ; 8$ voxels; $x=-36, y=6, z=24 ; Z=4.10 ; 6$ voxels). Temporal activations were found in several areas of the left and right superior temporal gyrus $(x=-66, y=-42$, $z=15 ; Z=4.80 ; 8$ voxels; $x=57, y=-39, z=9 ; Z=4.52$; 15 voxels, $x=-39, y=-54, z=3 ; Z=4.63 ; 9$ voxels) and left lateral occipito-temporal sulcus $(x=42, y=-51$, $z=-21 ; Z=4.41 ; 6$ voxels). Note the absence of activity in the left intraparietal sulcus and angular gyrus in both nicotine conditions.

A direct comparison between nicotine and placebo conditions, testing for increased reorienting-related activations under nicotine, yielded reorienting by drug interactions in several brain regions outside the parietal cortex, none of which showed a neural validity effect in a prior study without drug challenge (Thiel et al, 2004). The only parietal region exhibiting enhanced responses to nicotine was anterior to the intraparietal sulcus (see Table 3). The interaction in this anterior parietal region was due to both increased responses to invalid as compared to valid trials under nicotine and higher responses to valid as compared to invalid trials under placebo. The opposite contrast, which 
Table 3 Higher Activations under Nicotine as Compared to Placebo for Reorienting

\begin{tabular}{|c|c|c|c|c|c|c|}
\hline Region & Side & $x$ & $y$ & $\mathbf{z}$ & Voxels & Z-score \\
\hline \multicolumn{7}{|l|}{ Parietal } \\
\hline Ant. parietal & $\mathrm{L}$ & -27 & -42 & 39 & 18 & 5.67 \\
\hline \multicolumn{7}{|l|}{ Frontal } \\
\hline \multirow[t]{2}{*}{ Precentral gyrus/precentral sulcus } & L & -30 & -9 & 42 & 23 & 5.00 \\
\hline & $\mathrm{L}$ & -36 & 6 & 24 & 20 & 4.99 \\
\hline Cingulate sulcus & $\mathrm{R}$ & 9 & 9 & 54 & 11 & 4.91 \\
\hline \multicolumn{7}{|l|}{ Occipital } \\
\hline Fusiform gyrus & $\mathrm{R}$ & 30 & -48 & -24 & II & 5.11 \\
\hline Lat. Occ. sulcus & $\mathrm{L}$ & -39 & -54 & 6 & 17 & 4.51 \\
\hline \multicolumn{7}{|l|}{ Temporal } \\
\hline Insula & $\mathrm{L}$ & -45 & 3 & 3 & 14 & 4.35 \\
\hline \multicolumn{7}{|l|}{ Subcortical } \\
\hline Brainstem & L & -9 & -18 & -18 & 15 & 4.48 \\
\hline \multirow[t]{2}{*}{ Cerebellum } & L & -3 & -72 & -24 & 7 & 4.52 \\
\hline & $R$ & 9 & -57 & -33 & 8 & 4.15 \\
\hline
\end{tabular}

captures reduced neural activations under nicotine during reorienting, revealed significant effects in left intraparietal sulcus/angular gyrus, supramarginal gyrus, and precuneus after nicotine (see Figure 6 and Table 4). The right intraparietal sulcus activation did not exceed the voxel threshold of five contiguous voxels. That is, nicotine reduced the neural activity in mainly left parietal regions. Less activity was also found in the bilateral inferior occipital sulcus extending into the fusiform gyrus, in the right lingual gyrus, and in the cerebellum. The right parts of Figure $6 \mathrm{~b}$ illustrate the drug-induced differences in the left intraparietal sulcus in showing that the lack of a neural validity effect in the parietal cortex under nicotine is mainly driven by reduced neural activity in invalid trials. Note that a similar pattern was observed in the right intraparietal sulcus.

Given the apparent differences between the two doses of nicotine at our applied threshold (Figure 5), we further tested for dose-related effects by comparing reorientingrelated activity under 1 and $2 \mathrm{mg}$ of nicotine. The $2 \mathrm{mg}$ dose significantly increased reorienting-related activity as compared to $1 \mathrm{mg}$ in right cerebellum $(x=3, y=-54, z=-9$; $Z=5.26 ; 61$ voxels; $x=18, y=-72, z=-36 ; Z=4.75 ; 8$ voxels) and in the left superior temporal sulcus $(x=-66$, $y=-42, z=15 ; Z=4.45 ; 10$ voxels). The only region more active under $1 \mathrm{mg}$ of nicotine was the left cerebellum $(x=-36, y=-51, z=-42 ; Z=4.50 ; 10$ voxels). Note that the effects in intraparietal sulcus and precuneus shown in Figure 6 were not dose dependent (even when less conservative statistical testing, such as that on the parameter estimates, was performed). a
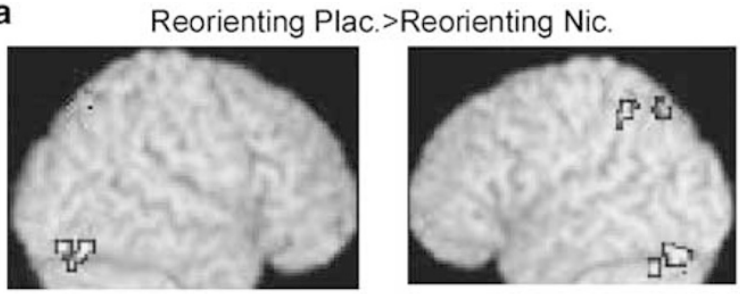

b
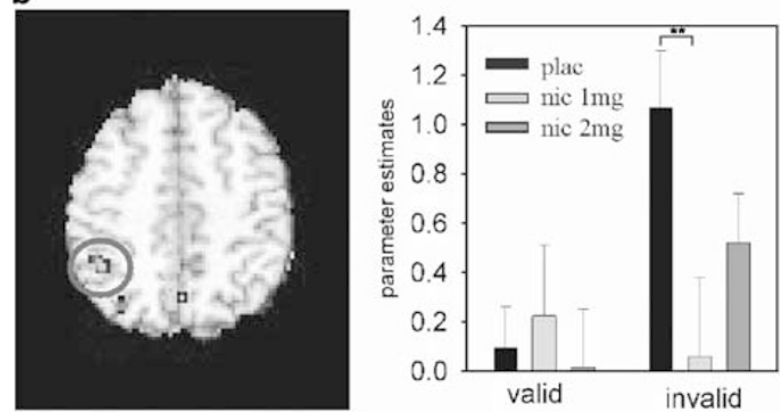

Figure 6 Reorienting by drug interaction. (a) Brain regions showing higher responses to invalid as compared to valid cue trials under placebo as compared to nicotine (see also Table 4). Note the significantly increased activity in the left intraparietal sulcus/angular gyrus, supramarginal gyrus, and precuneus. (b) Right intraparietal sulcus activation displayed in transverse section with plot of effect size (mean and SEM) from the maximum of the circled region as a function of trial type and drug. ${ }^{*} * p<0.01$ (post hoc Tukey's tests).

Table 4 Higher Activations under Placebo as Compared to Nicotine for Reorienting

\begin{tabular}{|c|c|c|c|c|c|c|}
\hline Region & Side & $x$ & $y$ & $\mathbf{z}$ & Voxels & Z-score \\
\hline \multicolumn{7}{|l|}{ Parietal } \\
\hline Intraparietal sulcus/angular gyrus & L & -45 & -48 & 51 & 16 & 4.52 \\
\hline Supramarginal gyrus & L & -33 & -69 & 51 & 9 & 4.18 \\
\hline Precuneus & & 0 & -66 & 45 & 7 & 4.06 \\
\hline \multicolumn{7}{|l|}{ Occipital } \\
\hline \multirow[t]{2}{*}{ Inf. occ. sulcus/fusiform gyrus } & $\mathrm{R}$ & 39 & -69 & -27 & 21 & 5.08 \\
\hline & L & -36 & -81 & -30 & 18 & 4.93 \\
\hline Lingual gyrus & $\mathrm{R}$ & 12 & -84 & -27 & 7 & 4.21 \\
\hline \multirow[t]{2}{*}{ Cerebellum } & & 0 & -78 & -42 & 7 & 4.22 \\
\hline & L & -30 & -63 & -39 & 8 & 4.58 \\
\hline
\end{tabular}

\section{DISCUSSION}

We investigated nicotinic modulation of brain systems involved in alerting and reorienting of visuospatial attention. Behaviorally, there was a trend for nicotine affecting reorienting. The fMRI data revealed the neural effects of nicotine on both attentional systems, which was, among others, evident in left parietal cortex during reorienting and in right parietal and frontal areas during alerting. 


\section{Behavioral Data}

A large body of evidence suggests that nicotine can improve measures of attention in smokers (Wesnes and Warburton, 1983; Parrott and Craig, 1992; Leigh et al, 1977; Hasenfratz and Battig, 1992; Rodway et al, 2000). Attentional improvements after nicotine administration in nonsmokers are, however, controversial (Heishman et al, 1993; Heishman and Henningfield, 2000; Foulds et al, 1996; Levin et al, 1998).

Our behavioral data suggest that nicotine may improve reorienting of attention in nonsmokers. Enhanced attentional reorienting after nicotine is in line with prior behavioral evidence in animals and smoking subjects (Witte et al, 1997; Stewart et al, 2001; Phillips et al, 2000; Murphy and Klein, 1998; Shirtcliff and Marrocco, 2003). The present data extend these prior findings in showing that cholinergic modulation of reorienting is observable not only in smokers but also in nonsmoking subjects, even though effects are small.

Another observation is that the behavioral effects of nicotine on reorienting were only seen in those subjects who are slow in reorienting (ie have a big validity effect) under placebo. Performance-dependent benefits of drugs have been previously described for the dopaminergic system (Mattay et al, 2000), and might be linked to genotype (Mattay et al, 2003; Mehta et al, 2000). Thus, increases in activity of neuromodulatory systems such as ACh or dopamine seem to vary and depend on initial performance. Note that reports on performance-dependent drug effects obtained from median splits might be due to the phenomenon of a regression to the mean. Since alternative methods (eg Perkins, 1999) are statistically also problematic, we nevertheless choose to illustrate our findings with a median split in order to suggest the testable hypothesis that patients with parietal lesions and attentional deficits, who show impaired reorienting, might benefit from nicotine.

Prior studies investigating the dose-dependent effects of nicotine in attentional tasks have yielded variable results with linear, curvilinear or nonexistent dose-response relationships (for a review, see Mancuso et al, 1999). In the present study, we did not observe a dose-response relationship with regard to the validity effect. This might be due to the nicotine gum leading to more variable plasma nicotine levels than, for example, subcutaneous injections of the drug. Since plasma levels were not obtained in the present study, we cannot argue against such explanation. However, when looking at the physiological effects of nicotine, a linear dose-response relationship was found for pulse frequency, arguing against variable plasma levels. Another possibility is that the dose-response relationship may depend on initial performance. Figure $2 \mathrm{~b}$ demonstrates a linear dose-response relationship only in those subjects who show a big validity effect under placebo. This underlines that subjects who benefit from nicotine show bigger effects with the higher dose.

\section{fMRI Data: Effects of Nicotine on Blood Flow}

A key issue in pharmacological fMRI studies is the effect of a drug on global and local blood flow or cerebrovascular coupling, which may confound the BOLD signal. Ghatan et al (1998), who investigated experimentally the effect of nicotine on global blood flow and cerebral oxygen uptake, found no effects of nicotine on these two measures. Furthermore, Jacobsen et al (2002), who measured the BOLD signal response in visual cortex to photic stimulation, showed that neither the height nor the extent of signal changed under nicotine, arguing against nicotine-induced alterations in cerebrovascular coupling. Similar results were found by Salmeron and Stein (2002) in motor cortex. Finally, it should be noted that pharmacological effects mediated through neurovascular coupling cannot explain the differential responses to specific stimuli such as invalid and valid trials for example.

\section{fMRI Data: Effects of Nicotine on Alerting and Reorienting of Attention}

Animal experiments indicate that the cholinergic innervation of parietal and frontal cortex is crucial for attentional processing (Bucci et al, 1998; Sarter et al, 2001). There are only view neuroimaging studies which have investigated the modulation of parietal and frontal activity with nicotine. Using a working memory task, Ernst et al (2001) found that nicotine gum enhanced memory-dependent neural activity in the prefrontal cortex and additionally activated bilateral parietal cortex in ex-smokers. Using a similar task and nonsmoking subjects, Kumari et al (2003) found activation increases in the left parietal and frontal areas and a decrease of right superior parietal activity in the working memory condition, with the highest memory load after subcutaneous nicotine. The only study investigating nicotinic modulation of attention-related brain activity was performed by Lawrence et al (2002) in smokers. Subjects were treated with a nicotine or placebo patch and performed a sustained attention task (rapid visual information-processing task). Nicotine treatment increased the number of hits and taskrelated BOLD activity bilaterally in the inferior and superior parietal cortex, precuneus, thalamus, and caudate nucleus, which indicates that the parietal cortex mediates nicotine's attention-enhancing effects during sustained attention.

Our selective attention task (cued target detection) enabled us to investigate the cholinergic modulation of subcomponents of visuospatial attention. Under placebo, we found alerting-related brain activity in extrastriate regions and reorienting-related activity in parietal and frontal brain areas, which is consistent with previous findings (Thiel et al, 2004). Nicotine differentially modulated alerting- and reorienting-related brain activity. Even though nicotine affected several brain regions including temporal, frontal, and occipital cortices, we restrict our interpretation to the effects of nicotine in the parietal cortex: first, because the parietal cortex is the brain region which has been hypothesized to contribute to shifting of attention (e.g. Corbetta et al, 2000; Thiel et al, 2004; Petersen et al, 1989; Robinson et al, 1995) and second because animal experiments suggest that this is the brain region where nicotine exerts its behavioral effects (Beane et al, 2002).

In order to understand the role of the parietal cortex and its modulation by nicotine in the present task, we first focus on the data of the placebo group. High parietal activity is 
found in two situations: in invalid trials, but also in no cue trials. Both cuing conditions involve targets appearing outside the current focus of attention. In invalid trials, attention is engaged in the opposite hemifield when targets occur. In no cue trials, attention is engaged at the fixation point when targets occur. This can be taken as indication that the parietal cortex is active whenever a target occurs outside the current focus of attention, which will induce enlargement of attentional focus and/or reorienting of attention. Conversely, parietal cortex activity is small when targets occur within the focus of attention, for example, in valid trials (see, however, Corbetta et al, 2000). The suggestion is also supported by data of two other fMRI studies. Using a similar paradigm, Small et al (2003) have shown that valid trials with a behavioral benefit (probably due to efficiently focusing attention on the cued region) induce less right and left parietal activation than valid trials with no behavioral benefit. Müller et al (2003) manipulated the size of the attended region by varying the number of cues and were able to demonstrate that lower activity is found in left intraparietal sulcus with a smaller focus of attention. There is also evidence from single-cell recordings in monkeys that some parietal cells, including neurons in area $7 \mathrm{a}$, intraparietal sulcus, and lateral parietal area, increase their firing rates in response to stimuli which occur outside the focus of attention, that is, invalid targets (Constantinidis and Steinmetz, 2001; Powell and Goldberg, 2000; Robinson et al, 1995).

The main action of nicotine in parietal brain regions is a stimulus-specific reduction of neural activity. When looking at BOLD activity to the different stimulus types under nicotine (Figure $4 \mathrm{~b}$ and $\mathrm{c}$ and $6 \mathrm{~b}$ and $\mathrm{c}$ ), it can be seen that the action of nicotine in the reorienting system results from an attenuated parietal activity in invalid trials and its action in the alerting system is due to a reduction of neural activity in no cue trials. Thus, under nicotine there is a reduction of neural activity to stimuli that occur outside the current focus of attention and need reorienting. Whether this action of nicotine is due to direct binding of the drug in parietal cortex or a modulation of parietal activity through binding elsewhere cannot be answered with fMRI.

In the following, we would like to speculate why nicotine should reduce parietal activity to 'unattended' stimuli. One reason why this might be the case is that subjects had a broader focus of attention under nicotine so that invalid targets were not 'unattended' and hence need less reorienting than under placebo. Indeed, there is behavioral and neural evidence to support this speculation. Studies of divided attention have shown that nicotine can enhance the ability to spread attention over multiple sources (Kassel, 1997). A broader focus of attention should speed detection of stimuli at unattended locations and might explain the decreased RTs in invalidly cued trials after nicotine. Recent fMRI evidence has further shown that increasing cholinergic function in nonsmokers with physostigmine reduced the degree of occipital lateralization of BOLD responses associated with directing attention to one hemifield. It is notable that this effect correlated with the detection of invalidly cued targets (Bentley et al, 2004). In other words, subjects who processed information on the invalidly cued side were faster to detect stimuli on that side. One effect of increasing cholinergic activity in the human brain might therefore be a decrease of top-down modulation induced by cues. One might argue that such decrease of top-down modulation should reduce RTs to validly cued trials, which was not observed in our data. Our paradigm was, however, not ideal to test the 'costs' of a broader focus of attention since we used very salient stimuli which are easy to detect.

Our finding of decreased parietal activity with nicotine seems to contradict the results by Lawrence et al (2002), who found increases in attention-related parietal brain activity. This might be due to several differences in the study design. First, our subjects were nonsmokers and it was shown previously that neural effects of nicotine might differ between nonsmokers and smokers (Ernst et al, 2001). It is notable that decreases in parietal cortical activity after cholinergic stimulation were also found in a recent fMRI study by Bentley et al (2004), who subjected nonsmokers to physostigmine. Physostigmine increased attentional performance and, among others, decreased neural activity in the superior medial parietal cortex during maintenance of attention. Second, Lawrence et al (2002) used a sustained attention task, whereas we used a selective attention task. Since attentional functions are not a unity and behavioral effects of nicotine may differ depending upon the attentional function investigated, one can assume that drugs may modulate parietal cortex activity differentially depending on the task.

\section{Behavior-Brain Relationship}

Even though the effects of nicotine on alerting and reorienting systems might be both explained by a reduction of parietal activity to unattended targets in invalid and no cue trials, it is of interest to note that, behaviorally, only reorienting is affected by nicotine. The issue of how to interpret changes in BOLD signal in the absence of behavioral effects has been raised previously and there is evidence to assume that fMRI data might be more informative than behavioral data since changes in cognitive strategies or effort are not necessarily reflected in behavioral measures such as RTs (Wilkinson and Halligan, 2004; Fink et al, 2002). This is also seen in other studies using nicotine, which showed drug-related changes in the absence of behavioral effects (Ghatan et al, 1998; Ernst et al, 2001).

\section{ACKNOWLEDGEMENTS}

We thank B Elghahwagi, G Oefler and C Giessing for assistance with scanning and JN Shah and the MR group for expert technical support. This work was supported by the Volkswagenstiftung and the BMBF.

\section{REFERENCES}

Beane M, Drew A, Massey K, Marrocco R (2002). Infusion of cholinergic and noradrenergic agents into rat posterior parietal cortex modifies orienting and alerting components of covert visual attention, Program No 67413 Abstract Viewer/ Iternary Planner Washington, DC. Society for Neuroscience, 2002 Online.

Benowitz NL, Porchet H, Sheiner L, Jacob III P (1988). Nicotine absorption and cardiovascular effects with smokeless tobacco 
use: comparison with cigarettes and nicotine gum. Clin Pharmacol Ther 44: 23-28.

Bentley P, Husain M, Dolan RJ (2004). Effects of cholinergic enhancement on visual stimulation, spatial attention, and spatial working memory. Neuron 41: 969-982.

Bond A, Lader M (1974). The use of analogue scales in rating subjective feelings. Br J Med Psychol 47: 211-218.

Bucci DJ, Holland PC, Gallagher M (1998). Removal of cholinergic input to rat posterior parietal cortex disrupts incremental processing of conditioned stimuli. J Neurosci 18: 8038-8046.

Clark CR, Geffen GM, Geffen LB (1989). Catecholamines and the covert orientation of attention in humans. Neuropsychologia 27: 131-139.

Constantinidis C, Steinmetz MA (2001). Neuronal responses in area $7 \mathrm{a}$ to multiple stimulus displays: II. Responses are suppressed at the cued location. Cereb Cortex 11: 592-597.

Corbetta M, Kincade JM, Ollinger JM, McAvoy MP, Shulman GL (2000). Voluntary orienting is dissociated from target detection in human posterior parietal cortex. Nat Neurosci 3: 292-297.

Coull JT, Nobre AC, Frith CD (2001). The noradrenergic alpha2 agonist clonidine modulates behavioural and neuroanatomical correlates of human attentional orienting and alerting. Cereb Cortex 11: 73-84.

Ernst M, Matochik JA, Heishman SJ, Van Horn JD, Jons PH, Henningfield JE et al (2001). Effect of nicotine on brain activation during performance of a working memory task. Proc Natl Acad Sci USA 98: 4728-4733.

Fan J, McCandliss BD, Sommer T, Raz A, Posner MI (2002). Testing the efficiency and independence of attentional networks. J Cogn Neurosci 14: 340-347.

Fink GR, Marshall JC, Weiss PH, Toni I, Zilles K (2002). Task instructions influence the cognitive strategies involved in line bisection judgements: evidence from modulated neural mechanisms revealed by fMRI. Neuropsychologia 40: 119-130.

Foulds J, Stapleton J, Swettenham J, Bell N, McSorley K, Russell MA (1996). Cognitive performance effects of subcutaneous nicotine in smokers and never-smokers. Psychopharmacology (Berl) 127: 31-38.

Friston KJ, Holmes AP, Worsley KJ, Poline J-P, Frith CD, Frackowiak RS (1995). Statistical parametric maps in functional imaging: a general linear approach. Hum Brain Mapp 2: 189-210.

Ghatan PH, Ingvar M, Eriksson L, Stone-Elander S, Serrander M, Ekberg $\mathrm{K}$ et al (1998). Cerebral effects of nicotine during cognition in smokers and non-smokers. Psychopharmacology (Berl) 136: 179-189.

Griesar WS, Zajdel DP, Oken BS (2002). Nicotine effects on alertness and spatial attention in non-smokers. Nicotine Tob Res 4: $185-194$.

Hasenfratz M, Battig K (1992). Action profiles of smoking and caffeine: stroop effect, EEG, and peripheral physiology. Pharmacol Biochem Behav 42: 155-161.

Heishman SJ, Henningfield JE (2000). Tolerance to repeated nicotine administration on performance, subjective, and physiological responses in nonsmokers. Psychopharmacology (Berl) 152: 321-333.

Heishman SJ, Snyder FR, Henningfield JE (1993). Performance, subjective, and physiological effects of nicotine in non-smokers. Drug Alcohol Depend 34: 11-18.

Jacobsen LK, Gore JC, Skudlarski P, Lacadie CM, Jatlow P, Krystal $\mathrm{JH}$ (2002). Impact of intravenous nicotine on BOLD signal response to photic stimulation. Magn Reson Imaging 20: 141-145.

Josephs O, Henson RN (1999). Event-related functional magnetic resonance imaging: modelling, inference and optimization. Philos Trans R Soc Lond B Biol Sci 354: 1215-1228.

Kassel JD (1997). Smoking and attention: a review and reformulation of the stimulus-filter hypothesis. Clin Psychol Rev 17: $451-478$
Kumari V, Gray JA, ffytche DH, Mitterschiffthaler MT, Das M, Zachariah E et al (2003). Cognitive effects of nicotine in humans: an fMRI study. Neuroimage 19: 1002-1013.

Lawrence NS, Ross TJ, Stein EA (2002). Cognitive mechanisms of nicotine on visual attention. Neuron 36: 539-548.

Leigh G, Tong JE, Campbell JA (1977). Effects of ethanol and tobacco on divided attention. J Stud Alcohol 38: 1233-1239.

Levin ED, Conners CK, Silva D, Hinton SC, Meck WH, March J et al (1998). Transdermal nicotine effects on attention. Psychopharmacology (Berl) 140: 135-141.

Mancuso G, Andres P, Ansseau M, Tirelli E (1999). Effects of nicotine administered via a transdermal delivery system on vigilance: a repeated measure study. Psychopharmacology (Berl) 142: 18-23.

Mattay VS, Callicott JH, Bertolino A, Heaton I, Frank JA, Coppola $\mathrm{R}$ et al (2000). Effects of dextroamphetamine on cognitive performance and cortical activation. Neuroimage 12: 268-275.

Mattay VS, Goldberg TE, Fera F, Hariri AR, Tessitore A, Egan MF et al (2003). Catechol $O$-methyltransferase val158-met genotype and individual variation in the brain response to amphetamine. Proc Natl Acad Sci USA 100: 6186-6191.

Mehta MA, Owen AM, Sahakian BJ, Mavaddat N, Pickard JD, Robbins TW (2000). Methylphenidate enhances working memory by modulating discrete frontal and parietal lobe regions in the human brain. J Neurosci 20: RC65.

Müller NG, Donner TH, Bartelt OA, Brandt SA, Villringer A, Kleinschmidt A (2003). The functional neuroanatomy of visual conjunction search: a parametric fMRI study. Neuroimage 20: $1578-1590$.

Murphy FC, Klein RM (1998). The effects of nicotine on spatial and non-spatial expectancies in a covert orienting task. Neuropsychologia 36: 1103-1114.

Parrott AC, Craig D (1992). Cigarette smoking and nicotine gum (0, 2 and $4 \mathrm{mg}$ ): effects upon four visual attention tasks. Neuropsychobiology 25: 34-43.

Perkins KA (1999). Baseline-dependency of nicotine effects: a review. Behav Pharmacol 10: 597-615.

Petersen SE, Robinson DL, Currie JN (1989). Influences of lesions of parietal cortex on visual spatial attention in humans. Exp Brain Res 76: 267-280.

Phillips JM, McAlonan K, Robb WG, Brown VJ (2000). Cholinergic neurotransmission influences covert orientation of visuospatial attention in the rat. Psychopharmacology (Berl) 150: 112-116.

Posner MI (1980). Orienting of attention. Q J Exp Psychol 32: 3-25. Posner MI, Fan J (2004). Attention as an organ system. In: Neurobiology of Perception and Communication: From Synapse to Society. The IVth De Lange Conference. Cambridge University Press: Cambridge.

Powell KD, Goldberg ME (2000). Response of neurons in the lateral intraparietal area to a distractor flashed during the delay period of a memory-guided saccade. J Neurophysiol 84: 301-310.

Robinson DL, Bowman EM, Kertzman C (1995). Covert orienting of attention in macaques. II. Contributions of parietal cortex. J Neurophysiol 74: 698-712.

Rodway P, Dienes Z, Schepman A (2000). The effects of cigarette smoking on negative priming. Exp Clin Psychopharmacol 8: 104-111.

Rorden C, Brett M (2000). Stereotaxic display of brain lesions. Behav Neurol 12: 191-200.

Salmeron BJ, Stein EA (2002). Pharmacological applications of magnetic resonance imaging. Psychopharmacol Bull 36: 102-129.

Sarter M, Givens B, Bruno JP (2001). The cognitive neuroscience of sustained attention: where top-down meets bottom-up. Brain Res Brain Res Rev 35: 146-160.

Shirtcliff EA, Marrocco RT (2003). Salivary cotinine levels in human tobacco smokers predict the attentional validity effect size during smoking abstinence. Psychopharmacology (Berl) 166: $11-18$. 
Small DM, Gitelman DR, Gregory MD, Nobre AC, Parrish TB, Mesulam MM (2003). The posterior cingulate and medial prefrontal cortex mediate the anticipatory allocation of spatial attention. Neuroimage 18: 633-641.

Stewart C, Burke S, Marrocco R (2001). Cholinergic modulation of covert attention in the rat. Psychopharmacology (Berl) 155: 210-218.

Thiel CM, Zilles K, Fink GR (2004). Cerebral correlates of alerting, orienting and reorienting of visuospatial attention: an eventrelated fMRI study. Neuroimage 21: 318-328.

Voytko ML (1996). Cognitive functions of the basal forebrain cholinergic system in monkeys: memory or attention? Behav Brain Res 75: 13-25.

Wesnes K, Warburton DM (1983). Effects of smoking on rapid information processing performance. Neuropsychobiology 9: 223-229.

Wilkinson D, Halligan P (2004). The relevance of behavioural measures for functional-imaging studies of cognition. Nat Rev Neurosci 5: 67-73.

Witte EA, Davidson MC, Marrocco RT (1997). Effects of altering brain cholinergic activity on covert orienting of attention: comparison of monkey and human performance. Psychopharmacology (Berl) 132: 324-334.

Witte EA, Marrocco RT (1997). Alteration of brain noradrenergic activity in rhesus monkeys affects the alerting component of covert orienting. Psychopharmacology (Berl) 132: 315-323. 